\title{
Early stochastic star formation, nucleosynthesis, and chemical evolution in proto-galactic clouds
}

\author{
Grant Mathews* \\ Center for Astrophysics and JINA, Department of Physics, University of Notre Dame \\ E-mail: gmathews@nd.edu
}

\section{Lamya Saleh}

Department of Physics and Astronomy, Northwestern University

E-mail: l-saleh@northwestern.edu

\section{Timothy C. Beers}

JINA: Joint Institute for Nuclear Astrophysics, Dept. of Physics \& Astronomy, Michigan State

University

E-mail: beers@pa.msu.edu>

\begin{abstract}
We discuss the nucleosynthesis and stochastic evolution of proto-galactic clouds in a model which is motivated by cold dark matter simulations of hierarchical galaxy formation. We utilize SNinduced star-formation within a model that follows the evolution of chemical enrichment and energy input to the clouds by Type II and Type Ia supernovae. We include metallicity-dependent yields for all elements at all times, and include effects of finite stellar lifetimes. We derive the metallicity distribution functions for stars in the clouds, their age-metallicity relation, and relative elemental abundances for a number of alpha- and Fe-group elements. More massive clouds are only stable when one assumes an initial mass function that is not biased towards massive stars. The observed dispersion in $[\alpha / \mathrm{Fe}]$ at low $[\mathrm{Fe} / \mathrm{H}]$ tends to be too large unless yields from the most massive stars $\left(M \sim 35-40 \mathrm{M}_{\odot}\right)$ are suppressed. We also require mixing of protogalactic clouds to reduce the dispersion to the observed values. We estimate that $\sim 50 \%$ of the proto-galactic clouds were well mixed during the early star formation epoch. We also show that the observed large dispersion in some (e.g. neutron-capture) elements can be attributed to the fact that the rare events which produce these are prevented from adequate mixing.
\end{abstract}

International Symposium on Nuclear Astrophysics - Nuclei in the Cosmos - IX

25-30 June 2006

CERN

*Speaker.

$\dagger$ Work at the University of Notre Dame supported by the US Department of Energy under Nuclear Theory grant DE-FG02-95ER40934. Work at Michigan State University supported by grants AST 95-29454, AST 00-98549, and AST 00-98508. Work also supported in part by PHY 02-16783, Physics Frontier Centers/JINA: Joint Institute for Nuclear Astrophysics, awarded by the National Science Foundation. 


\section{Introduction}

The oldest and most metal-poor halo stars are believed to provide clues to the early epochs of Galaxy formation and evolution. Accordingly, many studies have been performed to identify trends in the chemical composition of halo stars and their connection to spatial and kinematic properties $[23,24,40,19,7,36,20,2,3,9,12,13,4,10]$

A dispersion in the abundances of heavy elements, varying from almost no dispersion for some alpha and iron-group elements, to more than two orders of magnitude for neutron-capture elements has been detected at very low metallicities. This has led to the suggestion that the lowestmetallicity stars were the result of mixing of the ejecta from single, or at most a few, supernovae, with a limited amount of gas in the parent clouds [5, 40, 25, 26, 31]. However, the small dispersion observed for $\mathrm{Mg} / \mathrm{Fe}$ [4] may indicate that more efficient mixing of different regions occurred for ejecta containing this element and perhaps other alpha elements as well $[9,10,13]$. The present work is an attempt to first establish the expected dispersion of various elemental ratios in the limit of little mixing among various early star-formation regions. Then, we deduce the required mixing timescale necessary to account for the lack of dispersion observed in some elements.

The most favored current theory for galaxy formation is based on a hierarchical clustering scenario in which the Galaxy is assembled from cold dark matter sub-galactic halos (e.g., [34]). These proto-galactic clumps originate from density fluctuations in the early universe that accreted primordial gas from their surroundings. Merging processes lead to larger structures, and ultimately, the Galaxy. Cold dark matter (CDM) models are consistent with the observed kinematics of the halo of the Galaxy $[11,14]$ and with the suggested hybrid formation scenario in which the inner halo may have formed by a coherent contraction, while the outer halo was formed by accretion of metal- poor fragments from nearby systems (e.g., [11]).

Chemical evolution models that have treated these early stages still lack a complete picture that includes all chemical and dynamical effects. They are not yet capable of providing a comprehensive picture that reproduces the scatter in observed abundances at low metallicities. Some models in the literature $[41,21,15,17,18,27,38,54,2,3,52,33,53]$ have been able to reproduce, at least partially, the inhomogeneities observed for elemental abundances through a stochastic evolution scenario. These are, however, often limited for example to the study of only a few neutron-capture elements, and/or metallicity independent yields, and/or instantaneous recycling. In the present paper we incorporate metallicity dependent stellar ejecta and finite stellar lifetimes.

The enrichment processes that took place in these first structures were influenced by the stochastic initial conditions in each cloud, e.g. the initial cloud mass. While smaller clouds are susceptible to destruction by tidal forces and energetic feedback and may experience shorter episodes of chemical evolution, larger structures may have continued to enrich the interstellar media (ISM) for longer periods of time and survived the merging process, contributing eventually to the formation of the disk.

\section{The Model}

In this work we utilize a stochastic chemical-evolution model [41] which incorporates the main features of hierarchical galaxy formation as suggested by cold dark matter simulations. Our 
model is based upon several assumptions. These are: 1) In the first zero-metallicity clouds, the first-generation stars are born as population III (Pop III), explode, and the clouds that survive are enriched by their expelled yields; 2) Star formation is induced by supernovae, mainly Type II (SNeII); 3) Each individual supernova is taken to form a shell, which expands with an average velocity and and triggers star formation with an average efficiency $\varepsilon$ over a timescale $\Delta t$; 4) The shells' mass is the sum of the whole SNII gas and the swept-up surroundings, and the metallicity is given by a complete mixture of both metal contents; and 5) As nucleosynthesis enrichment of the clouds proceeds some fraction of the clouds merge and mix

The form for the SN-induced star formation assumes [54] that the material from which the next generation of stars forms is the result of complete mixing of the SN ejecta with the gas swept up from the ISM by the explosion. This produces low-metallicity stars with elemental abundances resembling the ejecta of high-mass SN progenitors, as suggested by the data.

We show that this simple stochastic model is capable of reproducing many features of the observed stallar abundances, relative to iron. The metallicity-dependent chemical yields of [55] used in this model, have been used previously in detail by [49]. We test these yields with our stochastic model for alpha and several iron-group elements down to $[\mathrm{Fe} / \mathrm{H}] \underset{<}{\sim}-4.0$. We note, however, that these yields were based upon scaled solar metallicities and are therefore not completely consistent with the evolved metallicities of the proto-galactic clouds computed here. This introduces uncertainty for some elements.

Our model consists of evolving individual clouds in the mass range of $10^{5}$ to $10^{8} \mathrm{M} \odot$. We follow the chemical evolution of each cloud as a one-zone closed box [50]. The gas and stars are then explicitly evolved in time. We assume that all halo field stars were formed originally in these proto-Galactic clouds and were later dispersed by tidal forces. By varying the total masses and the star formation histories of these clouds, we attempt to account for the trends and scatter in elemental abundances observed in metal-poor stars of the halo and thick-disk populations.

Simulations show that as the baryonic component of these clouds cools (mostly via molecular hydrogen) they gravitate to the center of the dark-matter potential to form central cores [1,30]. These cores are believed to form stars at their centers, the exact nature of which is still uncertain [44, 8]. We assume that each cloud starts with primordial material, and ultimately forms one massive star at its center. This Pop-III star later explodes as a SN event, triggering the formation of higher metallicity (population II) stars in its high-density shell. Subsequent star formation progresses in the shells of later SN events.

For a given cloud, when the first shell forms at $t=0$, the rate at which mass goes into stars is

$$
\psi(t=0)=\varepsilon M_{s h}(m, 0) / \Delta t
$$

where $\varepsilon$ is the star formation efficiency. In the present work, $\varepsilon$ was adjusted to reproduce the shift in slope of the elemental abundance ratios for three iron-group elements below $[\mathrm{Fe} / \mathrm{H}] \sim-2.4$ observed by McWilliam et al. [24]. It was also made to be consistent with the observation that the age-metallicity distribution changes for extremely metal- poor stars with $[\mathrm{Fe} / \mathrm{H}]<-2.4 . M_{s h}(m, t)$ is the mass of the shell formed in a SN explosion at time $t$, with the progenitor mass being $m$, and is given by :

$$
M_{s h}(m, t)=E_{j}(m, Z)+M_{s w},
$$


where $M_{s w}$ is the mass of the interstellar gas swept up by the expansion. Since the explosion energy of a core-collapse supernova depends only weakly on the progenitor mass [55, 48], this quantity is taken to be constant with a value of $5 \times 10^{4} \mathrm{M}_{\odot}[40,43,54] . E_{j}(m, Z)$ is the mass of all the ejected material from the SN with a progenitor mass $m$ and metallicity $Z$. For the first SN event the metallicity $Z$ is zero for a population III star, and $m$ is $m_{1}$. For later generations, the metallicity of a star is calculated from the metallicity of the shell from which it was formed, $Z_{s h}(m, t)$. This is calculated as a function of time, and is given by:

$$
Z_{s h}(m, t)=1-\left[x_{s h}^{H}(m, t)+x_{s h}^{H e}(m, t)\right] .
$$

Here, $x_{s h}^{H}$ and $x_{s h}^{H e}$ are the fractions of $\mathrm{H}$ and $\mathrm{He}$ in the shell, respectively, and are given by:

$$
\begin{aligned}
x_{s h}^{H}(m, t) & =\left[y^{H}(m, t)+x_{\text {gas }}^{H}(t) M_{s w}\right] / M_{s h}(m, t), \\
x_{s h}^{H e}(m, t) & =\left[y^{H e}(m, t)+x_{\text {gas }}^{H e}(t) M_{s w}\right] / M_{s h}(m, t),
\end{aligned}
$$

where $y^{H}$ and $y^{H e}$ are the mass of ejected $\mathrm{H}$ and $\mathrm{He}$, respectively, from the explosion. The quantities $x_{\text {gas }}^{H}$ and $x_{\text {gas }}^{H e}$ are the fractions of $\mathrm{H}$ and $\mathrm{He}$ in the interstellar gas at the time of formation of the shell $t$, respectively. The yields of individual elements, $y_{i}$ are given by

$$
\left\langle y_{i}(m, t)\right\rangle=\int_{\max \left\{m\left(t-\tau_{m}\right), 10\right\}}^{m_{u}} d m^{\prime} u\left[\phi\left(m^{\prime}\right) / m^{\prime}\right] \times y_{i}^{e j}\left(m, Z_{s h}\left(m^{\prime}, t-\tau_{m}\right)\right),
$$

where $y_{i}^{e j}\left(m, Z_{s h}\left(m^{\prime}, t-\tau_{m}\right)\right)$ is the mass of the element $i$ ejected from the star with progenitor mass $m$ and metallicity $Z_{s h}\left(m^{\prime}, t-\tau_{m}\right)$. The change in gas mass with time is then given by:

$$
\frac{d M_{g}}{d t}=-\psi(t)+\int_{\max \left(m_{t}, m_{l}\right)}^{m_{u}} d m[\phi(m) / m] \times M_{e j}(m, t) \psi\left(t-\tau_{m}\right) .
$$

We utilize the stellar yields calculated by [55] for high- mass stars. The uncertainties associated with the use of such supernova yields have been critically summarized in $[2,3]$. In particular, the synthesized yields can be sensitive to various aspects of both the stellar nucleosynthesis models and the supernova explosion mechanism and energy released. For intermediate stellar masses we adopt the standard nucleosynthesis yields of Renzini and Voli [39]. Although other tracks are available (e.g. [35]), the tracks of [39] are sufficient for the present study, which is almost unaffected by the yields of intermediate mass stars other than through the overall evolution of metallicity. Intermediate-mass stars mostly end their lives as carbon-oxygen rich white dwarfs. If they are members of a binary system these dwarfs may accrete mass from the remaining member to the point where their mass exceeds the Chandrasekhar limit. At this point, the star becomes unstable, causing a thermonuclear Type-Ia SN event. For these events we use the yields calculated by [47].

The baryonic masses of globular clusters observed in the Galaxy range between $10^{4}$ and $10^{6}$ $\mathrm{M}_{\odot}$, while the minimum total mass of dwarf galaxies in the Local Group are $\sim 2 \times 10^{7} \mathrm{M}_{\odot}$ [22]. Therefore, we choose initial total cloud masses in the range $\left(10^{5}-10^{8}\right) \mathrm{M}_{\odot}$. In this mass range, the clouds are sensitive to stellar feedback, both in terms of chemical enrichment and energetics. We start with gas in a single phase and uniform density, and choose the volume of each cloud such that we maintain a reasonable initial density in the clouds $\left(\sim 0.25\right.$ particles $\left.\mathrm{cm}^{-3}\right)$ corresponding to a baryon surface density of about $0.01 \mathrm{M}_{\odot} \mathrm{pc}^{-2}$. This condition is necessary in order to calculate the initial potential energy of the cloud, and its lifetime prior to destruction by SN energy input. 

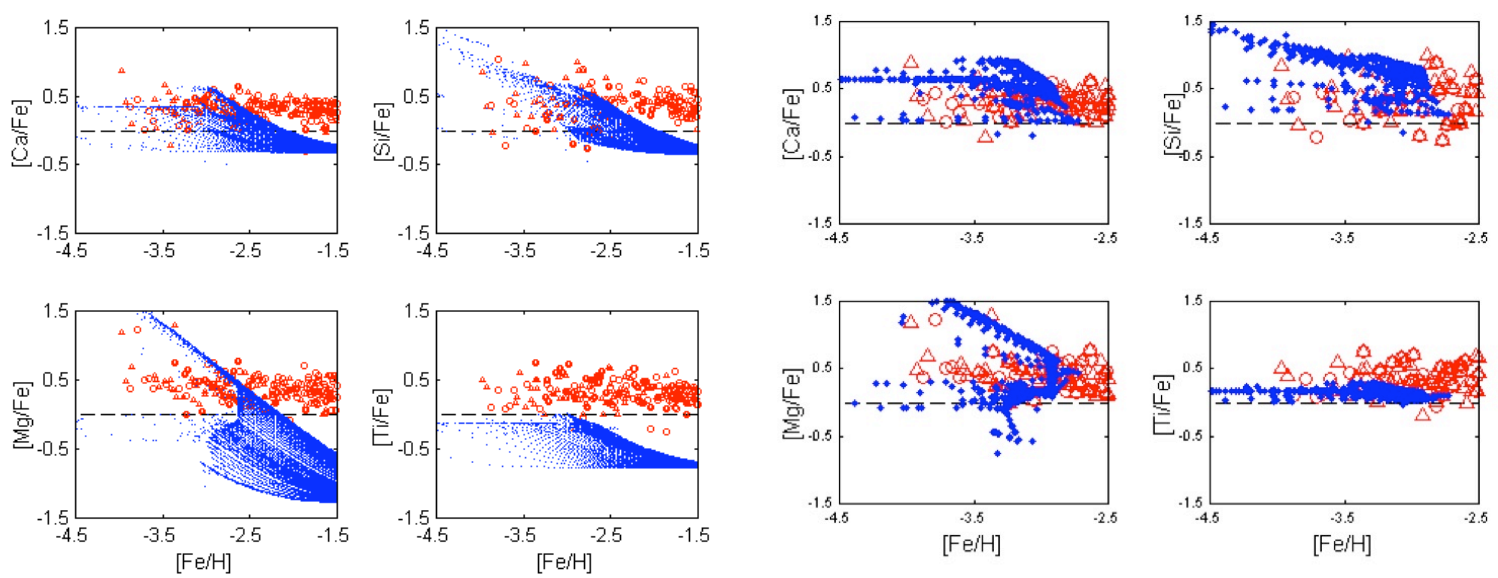

Figure 1: Comparison of model and observed abundances of the alpha elements $\mathrm{Mg}, \mathrm{Ca}, \mathrm{Si}$, and Ti. Data is from Ryan et al. (1996) (triangles) and Norris et al. (2001) (circles). The small (dots) represent the model stars. The two right panels show abundances of the alpha elements when Fe yields are reduced by a factor of two,.

\section{Results}

Simulations were run for $5 \mathrm{Gyr}$ using a Salpeter initial mass function. After $1 \mathrm{Gyr}$, SN of typeIa were allowed to contribute their ejecta. The elemental abundances were calculated and recorded for every shell that forms during the simulation. The model produces a small number of stars at very low metallicities, $[\mathrm{Fe} / \mathrm{H}]<-3$, and without mixing of the clouds, shows a considerable spread in $[\mathrm{X} / \mathrm{Fe}]$ ratios. The scatter of the abundances for model stars is given by the spread in metallicities of the SN models .

The left side of Figure 1 shows $[\alpha / \mathrm{Fe}]$ vs. $[\mathrm{Fe} / \mathrm{H}]$ for several alpha elements calculated by a straightforward application of the model (small dots). These are compared with observational data of [40,32]. There are two problems with a naive application of the model. 1) The observed abundance plateau at low metallicities for alpha-elements is not achieved; and 2) The narrow observed dispersion of alpha elements [4, 6, 10, 13, 9] is not obtained; 3) There is also a peculiar trend in the data whereby some stars which appear to have increasing $[\alpha / \mathrm{Fe}]$ with decreasing $[\mathrm{Fe} / \mathrm{H}]$ which shows up as an extension in the data. This behaviors is apparent in the abundances of $\mathrm{Mg}, \mathrm{Ca}$, and $\mathrm{Si}$. On the other hand, Ti shows a scatter that is almost constant over the metallicity range.

Regarding the over-abundance plateau in alpha elements, this must result from the early dominance of SNeII. The problem, however, is that the precise mass-cut in supernovae is not known. Indeed, we are free to place it where we wish. We can recover the plateau therefore by simply reducing the iron yields from the stellar models. The right two panels of Figure 1 show the results of our model when the Fe yield is reduced by a factor of 2 . The agreement between the data and the calculation is much improved. There are two trends in the data particularly apparent for $\mathrm{Mg}$. One is a flattening at $[\mathrm{X} / \mathrm{Fe}] \sim 0.5$, and another a linear increasing band in $[\mathrm{X} / \mathrm{Fe}]$ with decreasing metallicity. 

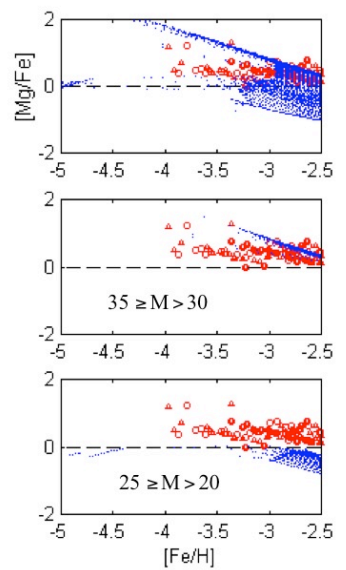
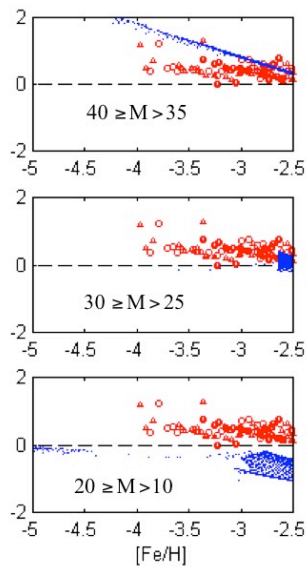
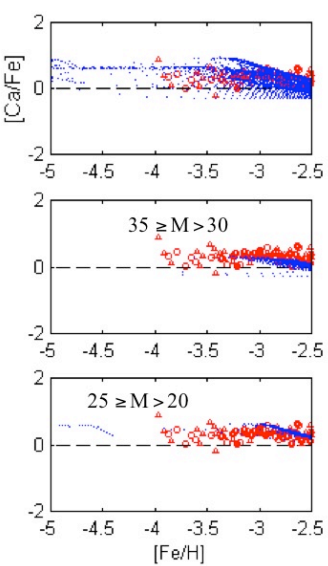
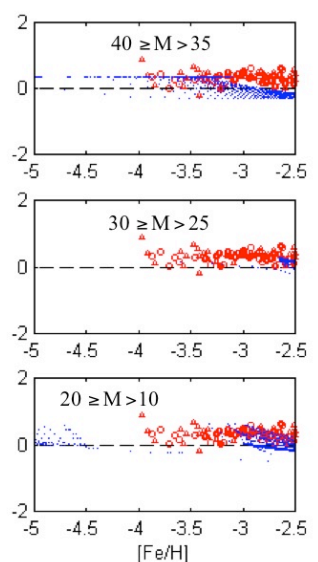

Figure 2: Contribution (small dots) from different progenitor masses to $[\mathrm{Mg} / \mathrm{Fe}]$. (2 left panels) and $[\mathrm{Ca} / \mathrm{Fe}]$ (two right panels). The top left figure shows the whole range. Data is from Ryan et al. (1996) (triangles) and Norris et al. (2001) (circles).

Regarding the narrow band of elements with high $[\mathrm{Mg} / \mathrm{Fe}]$, these stars were affected by the ejecta of the most massive stars. Figure 2 shows the contributions to the elemental abundances by different progenitor masses down to metallicities of $[\mathrm{Fe} / \mathrm{H}]=-5$ and up to -2.5 . This range represents the early stages of chemical evolution in the Galaxy for which our model is applicable. Once $[\mathrm{Fe} / \mathrm{H}]>-2.5$, the contributions from SNIa pollute the ISM and the contributions from SN of Type II become less dominant. For these figures, we have run the simulation allowing only a range of progenitor masses to contribute its ejecta. The mass ranges are shown on the figures. Here it is apparent that this band is produced by massive stars $\left(35 \mathrm{M}_{\odot}<M<40 \mathrm{M}_{\odot}\right)$. Since very few such stars are actually observed we conclude that the early halo may not have been contaminated by their ejecta. Rather, these stars may have collapsed into black holes.

Even after reducing the effective range of the stars which eject synthesized material we find that the dispersion in the $[\alpha / \mathrm{Fe}]$ ratios are too large without introducing mixing. For example, for $[\mathrm{Mg} / \mathrm{Fe}]$ the dispersion near $[\mathrm{Fe} / \mathrm{H}] \sim-3$ reduces from $\sigma=0.35$ to 0.20 when the most massive stars are removed. This is still much more than the reported [10] dispersion of $\sigma \leq 0.10$. We can however use this discrepancy with the observed dispersion to constrain $[3,14]$ the degree of mixing of super nova remnants in the early ISM.

It is easy to show that the mixing of proto-galactic clouds in our model can explain the smaller observed dispersion. The effects of metallicity on the observed dispersion can be related to an efficiency parameter $\varepsilon_{m i x}$ :

$$
\sigma_{o b s}=\sigma_{\text {clouds }}\left(1-\varepsilon_{\text {mix }}\right)
$$

The difference between the observed and calculated dispersion, therefore, will require a mixing efficiency of about $50 \%$.

Even though the alpha elements have a narrow dispersion one must still explain the broad dispersion seen is other elements such as the neutron capture elements [45]. This can be attributed to the fact that these elements are producuced in rare events. This modifies the mixing efficiency 
for species $i$ by the fraction of stars which contribute to its production. For example, if only stars in a narrow mass range $m_{l}$ to $m_{u}$ contribute then the mixing efficiency becomes:

$$
\varepsilon_{e f f}=\varepsilon_{m i x}\left[\frac{\int_{m_{l}}^{m_{u}} m \phi(m) d m}{\int_{0}^{\infty} m \phi(m) d m}\right] .
$$

If the $r$-process elements are only produced in a narrow range of stars, say $m=10-11 \mathrm{M}_{\odot}$, then the mixing efficiency reduces to about $2 \%$. Basically, even though every cloud has alpha elements, not all have r-process ejecta so that even after half of the clouds mix a large dispersion of $r$-process elements survives.

We have also calculated [41] values of relative abundances for $\mathrm{Cr}$, Co, $\mathrm{Mn}$ and Ni. The observed ratios of $\mathrm{Mn}$ and of $\mathrm{Ni}$ are reproduced well by the metallicity- dependent yields of [55] in this model. Cr, on the other hand, is diffiult to reproduce as others have found [3]. The stars produced at very low metallicity show a small over-abundance relative to solar, contrary to the observed under-abundance. The excess in $\mathrm{Co} / \mathrm{Fe}$ was produced fairly well with the metallicitydependent yields below $[\mathrm{Fe} / \mathrm{H}]=-2.5$ if the contributions from $\mathrm{SN}$ more massive than $40 \mathrm{M}_{\odot}$ are excluded. This behavior of Co at the lowest metallicities has not been produced by any previous models, and no known stellar yields were able to predict it even by modifications in explosion parameters of SN-II models (e.g. [3, 28]).

\section{Conclusions}

There are a number of conclusions to be drawn from this study. The first is that a good understanding of iron ejection in supernovae is needed to quantitatively reproduce the observed $[\mathrm{X} / \mathrm{Fe}]$ rations. This will require eventual multidimensional models of the mixing and mass cut near the iron core in supernova simulations.

A second conclusion is that the absence of a band of stars with high $[\mathrm{Mg} / \mathrm{Fe}]$ ratios suggests that the most massive stars $\left(m_{\sim} 35 \mathrm{M}_{\odot}\right)$ may not contribute to nucleosynthesis enrichment in the early galaxy, but instead collapse to black holes.

The third conclusion is that the observed narrow dispersion among alpha elements requires significant mixing of the proto-galactic clouds during this early nucleosynthesis epoch. We estimate that a mixing efficiency of about $>50 \%$ is needed to account for the narrow dispersion in the alpha elements.

The fourth conclusion is that even though the alpha elements appear well mixed, it is possible to obtain the broad dispersion seen in neutron capture and other elements as a consequence of their production arising from rare events. If only a narrow range of stars contributes to the $r$ process (e.g. 10-11 $\left.\mathrm{M}_{\odot}\right)$ then only a small fraction of clouds $(\sim 2 \%)$ will be able to mix so that the dispersion within the isolated clouds remains even if half of the clouds mix.

Finally, iron group elements $\mathrm{Cr}, \mathrm{Co}, \mathrm{Mn}$, and $\mathrm{Ni}$ all exhibit peculiar abundance trends vs. $[\mathrm{Fe} / \mathrm{H}]$ than those predicted by the model. This is particularly hard to explain as such elements are expected to be ejected along with iron. One possible explanation may be is to move the mass-cut for iron and iron-group elements outward in the stellar models for all stars except those with masses in the range $25<m<35 \mathrm{M}_{\odot}$. If yields are restricted in this way a good fit to all elements can be 
obtained. We note, however, that ejecta from hypwenovae may be required to boost the yield of these elements at low metallicity.

Overall, this study has shown the importance of modeling the nucleosynthesis and evolution of the elements in the context of current understanding of how the early galaxy forms [37]. Clearly, however, much more work need to be done before a satisfactory understanding is obtained. For example, the dependence of the results on the detailed stellar nucleosynthesis [16] is valuable.

\section{References}

[1] Abel, T., Bryan, G.L., \& Norman, M. 2002, Science, 295, 93

[2] Argast, D., Samland, M., Gerhard, O.E., Thielemann, F.-K. 2000, A\&A, 356, 873

[3] Argast, D., Samland, M., Thielemann, F.-K. \& Gerhard, O.E. 2002, A\&A, 388, 842

[4] Arnone, E. Ryan, S. G., Argast, D. Norris, J. E. \& Beers, T. C. 2004, Astron. Astrophys, 430, 507

[5] Audouze, \& Silk 1995, ApJ, 451, L49

[6] P. S. Barklem , N. Christlieb , T. C. Beers, V. Hill, M. S. Bessell , J. Holmberg, B. Marsteller, S. Rossi , F.-J. Zickgraf, , D. Reimers 2005, Astron. Astrophys., 439, 129

[7] Carney, B. W., Wright, J. S., Sneden, C., Laird, J. B., Aguilar, L. A., Latham, D.W. 1997, A J, 114, 363

[8] Carr, B. J., Bond, J. R., \& Arnett, W. D. 1984, ApJ, 277, 445

[9] Carretta, E., Gratton, R., Cohen, J.G., Beers, T.C., Christlieb, N. 2002, AJ, 124, 481

[10] Cayrel, R. et al. 2004, A\&A, 416, 1117

[11] Chiba, M., \& Beers, T.C. 2000, AJ, 119, 2843; Chiba, M., \& Beers, T.C. 2001, ApJ, 549, 325

[12] Cohen, J.G., Christlieb, N., Beers, T.C., Gratton, R., Carretta, E. 2002, AJ, 124, 470

[13] Cohen, J.G., C. Norbert, A. McWilliam, S. Stechman, I. Thompson, G. J. Wassweburg, I. Ivans, M. Dehn, T. Karlsson, J. Melendez 2004, ApJ, 612, 1107

[14] Font, A. S., Johnston, K. V., Bullock, J. S., Robertson, B. 2006, ApJ, 638, 585

[15] Ishimaru, Y. \& Wanajo, S. 1999, ApJL, 511, L33

[16] Ishimaru, Y. Wanajo, S., \& Prantzos, N. (2006): this conference proceedings.

[17] Karlsson, T. and Gustafsson, B. 2005 Astron. Astrophys., 436, 879

[18] Karlsson, T. 2005 ApJ, 439, 93

[19] King, J.R. 1997, AJ, 113, 2302

[20] Kinman, T., Castelli, F., Cacciari, C., Bragaglia, A., Harmer, D, Valdes, F. 2000 A\&A, 364, 102

[21] Malinie, G. Hartmann, D.H., Clayton, D. D. \& Mathews, G.J. 1993, ApJ, 413, 633

[22] Mateo, M. 2000, The First Stars, Proc. Of the MPA/ESO Workshop, Garching, August 1999. Achim Weiss, Tom G. Abel, Vanessa Hill (eds.), Springer.

[23] Mathews, G.J., Bazan, G., Cowan, J.J. 1992, ApJ, 391, 719

[24] McWilliam, A., Preston, G. W., Sneden, C., Searle, L. 1995, AJ, 109, 2757 
[25] McWilliam, A. 1997, ARA\&A, 35, 503

[26] McWilliam, A. 1998, AJ, 115, 1640

[27] McWilliam, A., Searle, L. 1999, Ap\&SS, 265,133

[28] Nakamura, T., Umeda, H., Nomoto, K., Thielemann, F., Burrows, A. 1999, ApJ 517, 193

[29] Nomoto, K. Iwamoto, K., Kishimoto, N. 1997, Sci., 276, 1378

[30] Norman M. L., Abel T., Bryan G. 2000, "The First Stars", Proceedings of the MPA/ESO Workshop, Garching, Germany Eds. A. Weiss, T. Abel \& V. Hill, (Springer Verlag, Heidelberg), p. 250

[31] Norris, J.E., Beers, T.C., Ryan S.G 2000, ApJ, 540, 456

[32] Norris, J.E., Ryan, S.G., \& Beers, T.C. 2001, ApJ, 561, 1034

[33] Oey, M. S. 2003, MNRAS, 339, 849

[34] Peacock, J. 1999, Proc. of the MPA- ESO Cosmology Conference, Garching, Germany, Evolution of large scale structure : from recombination to Garching, edited by A. J. Banday, R. K. Sheth, L. N. da Costa. Garching, Germany : European Southern Observatory, p.64

[35] Portinari, L., Chiosi, C. \& Bressan, A. 1998, A\&A, 334, 505

[36] Preston, G. W. \& Sneden, C. 2000, AJ, 120, 1014

[37] Prantzos, N. (2006): this conference proceedings

[38] Raiteri, C. M., Villata, M., Gallino, R., Busso, M., Cravanzola, A. 1999, ApJ, 518, L91

[39] Renzini, A., \& Voli, M. 1981, A\&A, 94, 175

[40] Ryan, S. G.,Norris, J. E., \& Beers, T.C. 1996, ApJ, 471, 254

[41] Saleh, L., Beers, T. C. \& Mathews, G. J. 2006, J. Phys. G., 32581

[42] Sedov, L. 1946, Prikl. Mat. Mekh., 10(2) 241

[43] Shigeyama, T. \& Tsujimoto, T. 1998, ApJ, 507, L135

[44] Silk, J. 1983, MNRAS, 205,705

[45] Sneden, C., Cowan, J. J., Burris, D. L., Truran, J. W. 1998, ApJ,496, 235

[46] Steinmetz, M. 2003, A\&SS, 284, 325 (2003)

[47] Thielemann, F.-K., Nomoto, K., Yokoi, K. 1986, A\&A, 158,17

[48] Thielemann, F.-K., Nomoto, K., \& Hashimoto, M. 1996, ApJ, 460,408

[49] Timmes, F.X., Woosley, S.E., \& Weaver, T.A. 1995, ApJS, 98, 617

[50] Tinsley, B.M. 1980, Fundamentals of Cosmic Physics, 5, 287

[51] Tolstoy, E., Venn, K.A., Shetrone, M., Primas, F., Hill, V., Kaufer, A., Szeifert, T. 2003, AJ, 125, 707

[52] Travaglio, C., Burkert, A., Galli, D. 2000, The Galactic Halo : From Globular Clusters to Field Stars, Proceedings of the 35th Liege International Astrophysics Colloquium, Eds. A. Noels, P. Magain, D.

Caro, E. Jehin, G. Parmentier, and A. A. Thoul. Liege, Belgium : Institut d'Astrophysique et de Geophysique, p.135

[53] Tumlinson, J. 2006 Mon. Not. R. Astron. Soc., in press

[54] Tsujimoto, T., Shigeyama, T., \& Yoshii, Y. 1999, ApJ, 519, L63

[55] Woosley, S.E. \& Weaver, T.A. 1995, ApJS, 101, 181 (WW95) 